(2) if $0<\lim \inf |y| / x^{1 / 2}$ and lim sup $|y| / x^{1 / 2}<\infty$, then

$\Gamma(x+y+1, x) \sim\left(\frac{\pi}{2}\right)^{1 / 2} e^{y^{2} / 2 x-x} x^{x+y+1 / 2}\left[1+\frac{2}{\pi^{1 / 2}} \operatorname{Erf} \frac{y}{(2 x)^{1 / 2}}\right]$

(3) if $y>0$ and $x^{1 / 2}=o(y)$, then

$$
\Gamma(x+y+1, x) \sim\left(\frac{x+y}{e}\right)^{x+y}(2 \pi x)^{1 / 2} ;
$$

(4) if $y<0$ and $x^{1 / 2}=o(|y|)$, then

$$
\Gamma(x+y+1, x) \sim-\frac{1}{y} x^{x+y+1} e^{-x} .
$$

The University of Minnesota and

California Institute of Technology

\title{
A RESIDUE THEOREM FOR FINITE BLASCHKE PRODUCTS
}

\section{MAURICE HEINS}

1. Finite Blaschke products have long played a central role in the theory of bounded analytic functions, appearing frequently as functions enjoying various extremal properties. The present note is concerned with one such extremal property and its implications. Our principal result is:

If a finite Blaschke product has poles in the finite plane, then for at least one such pole the residue does not vanish.

Of course the presence of a simple pole in the finite plane renders the theorem trivial. However if one considers the case of a finite Blaschke product which is even and all of whose poles are multiple, then the existence of a nonzero residue is much more concealed. For the evenness of the Blaschke product would force the coefficient of $z^{-1}$ in its expansion at $\infty$ to vanish so that the presence of residues of the desired type would escape detection on examination of the residue at $\infty$; on the other hand a direct study of the residues corresponding to the finite poles, while not out of the realm of possibility, leads in the case of a large number of poles to a computational morass. A further complicating factor is revealed by examples ( $\$ 5)$ of finite Blaschke products with residue vanishing at some finite pole.

2. We recall that a finite Blaschke product is a rational function admitting a representation of the form

Received by the editors July 31, 1950. 


$$
b(z)=A z^{\nu_{0}} \prod_{1}^{n}\left(\frac{z-r^{2} / \bar{a}_{k}}{z-a_{k}}\right)^{\nu_{k}}
$$

where $A \neq 0, r>0,\left|a_{k}\right|>r(k=1, \cdots, n), \nu_{k}$ is a non-negative integer $(k=0, \cdots, n)$.

We assert that $b$ has the following extremal property: Let $f$ be meromorphic in $|z|>r$ and satisfy: (1) $\lim _{z \rightarrow \infty} f / z^{\nu} 0=A$, (2) $f$ has poles in the finite plane at most at the points $a_{k}$ and of orders not exceeding $\nu_{k}$ respectively. Then

$$
\limsup _{|z| \rightarrow r}\left|\frac{f}{b}\right| \geqq 1
$$

and equality holds if and only if $f=b$.

The proof is immediate from the maximum principle since $f / b$ is analytic in $|z|>r$ and takes the value one at $\infty$. This extremal property of course generalizes the well known result that a polynomial of degree $n$ with highest term having coefficient one has maximum modulus at least one on the unit circle and equality holds only for $z^{n}$.

3. Proof of theorem. Suppose that the assertion is false and that $b$ is a finite Blaschke product with finite poles all of which are residue free. Let $\beta$ denote the constant value of $\left|b\left(r e^{i \theta}\right)\right|$. Let $B$ denote the primitive of $b$ which vanishes at the origin and finally let

$$
b^{*}=\left(\nu_{0}+1\right) \frac{B}{z} .
$$

It is immediately verified that $b^{*}$ is an admitted $f$ in the sense of $\$ 2$. Further for $|z| \leqq r$, we have

$$
|b(z)| \leqq\left|\frac{z}{r}\right|^{\nu_{0}} \beta
$$

so that

$$
\left|b^{*}\left(r e^{i \theta}\right)\right| \leqq \frac{\nu_{0}+1}{r} \int_{0}^{r} \beta\left|\frac{z}{r}\right|^{\nu_{0}}|d z|=\beta .
$$

Hence $b^{*}=b$. The contradiction is apparent since the orders of the finite poles of $b^{*}$ are effectively less than the orders of the corresponding poles of $b$.

4. Our theorem admits application to the following problem: Do there exist finite Blaschke products with finite poles having the 
property that the derivative of some order (greater than or equal to 1) is also a finite Blaschke product? That the answer is always negative follows on noting that differentiation of a rational function yields a rational function with vanishing residues at all poles.

5. We have indicated that there exist finite Blaschke products (with at least two finite poles) whose residue at some finite pole vanishes. To construct an example of such a function let $0<a_{1}<1$ and consider

$$
\left(\frac{z-a_{1}}{1-a_{1} z}\right)^{2}\left(\frac{z-a_{2}}{1-a_{2} z}\right)^{2}
$$

where $a_{1}<a_{2}<1$. With $a_{1}$ fixed, it is easily verified that there exists an $a_{2}$ restricted as indicated such that the residue at $a_{1}^{-1}$ vanishes. The considerations are immediate and are omitted. The range of such examples can be further extended.

BROWN UNIVERSITY 